\title{
An Analysis and Design of a Virtual Collaboration Information System of the Jamu Supply Chain Network Based on a Fair Adaptive Contract
}

\author{
Aster Aryati Rakhmasari and Dian Anwar \\ Department of Electronic Industries Logistic Management, Polytechnic APP, \\ Jakarta, Indonesia
}

\begin{abstract}
Purpose - The purpose of this study is to analyze and design a virtual collaboration information system of the Jamu supply chain network based on a fair adaptive contract to respond to stability challenges in business with an uncertainty environment condition.

Design/Methodology/Approach - System entity approach is used for the purpose of system analysis and then case, sequence diagram, class diagram, graph data modeling, and business process modeling and Notation verse 2 (BPMN2.0) are also used to design the system concept. Each process of the connecting event and gateways was arranged with relevant multicriteria decision approach, whilst an adaptive quantitative formulation based on prescriptive analytics was embedded to ensure a fair contract implementation during the terms for all sphere roles in supply chain.
\end{abstract}

Findings - The results and implications of this model emphasizes on the knowledge, competence, trust, and fair (KCTF) for actors' adoption to improve supply chain stability and implementation.

Practical Implications - This led actors to collaborate their business in a more effective way.

Originality/Value - This model can reach more stability in the uncertainty environment.

Keywords Adaptive contract, Jamu agro-industrial supply chain, performance

All papers within this proceedings volume have been peer reviewed by the scientific committee of the Malikussaleh International Conference on Multidisciplinary Studies (MICoMS 2017).

\section{Introduction}

Jamu is a traditional herbal medicine. It has been practiced for many centuries in the Indonesian community to maintain good health and for treatment (Elfahmi et al., 2008). Contract design in the jamu supply chain under uncertainty environment is one of the prospective solutions to answer the jamu supply chain challenges. Existing contracts have not been able to respond to these challenges. According to Schroeder et al. (2011), the adaptive term in contract means the contract systems are capable of adapting their behavior

(C) Aster Aryati Rakhmasari, Dian Anwar. Published in the Emerald Reach Proceedings Series. Published by Emerald Publishing Limited. This article is published under the Creative Commons Attribution (CC BY 4.0) licence. Anyone may reproduce, distribute, translate and create derivative works of this article (for both commercial and non-commercial purposes), subject to full attribution to the original publication and authors. The full terms of this licence may be seen at http:// creativecommons.org/licences/by/4.0/legalcode 
Proceedings of to changes in their environment. In order to make a contract in a supply chain system MICoMS 2017 represent each entity/actor, we can use a virtual collaboration approach.

Virtual collaboration follows the same process as collaboration, but the parties involved in virtual collaboration do not physically interact and communicate exclusively through technological channels (Sutanto et al., 2011; Ho et al., 2013; Ho et al., 2016) develop adaptive contract design for crowdsourcing market (Schroeder et al., 2011). This paper analyzes and designs a virtual collaboration information system based on fair adaptive contract for jamu supply chain in order to respond stability challenges (Arshinder et al., 2011) in a supply chain with uncertainty environment condition.

\section{Methodology and result}

\subsection{The virtual collaboration information system analysis}

The requirement analysis of the virtual collaboration system based on fair adaptive contract is analyzed by system entity as the representation of the system (Wasson, 2015). System analysis is used to design a conceptual model of the system. The conceptual design is used to make the developer easier to understand behavior of the system.

System analysis results are virtual collaboration adaptive information system requirement to develop the system based on fair adaptive contract. Because, the goal of system is generating fair adaptive contract the main variables and parameters in the system analysis in both quantity and quality, and the other variables that common in supply chain. There are also cost and time as the support variables.

\subsection{Business process modeling, design use case, and sequence diagram}

In this research, Business Process Modelling Notation verse 2.0 (BPMN 2.0) is used to design the business process model. BPMN 2.0 is standard notation for business process modeling that widely applied by business analyst (Zur et al., 2013).

The next step of conceptual modeling design is to design the actor's role and their interaction/relationship in collaboration system by using use case diagram. Use case diagram is used to identify the different types of users of a system and the different use cases and will often be accompanied by other types of diagrams as well (Almutairi et al., 2013). So, the higher-level view of the system is easier to understand. Then, sequence diagram is used to detail the sequence of each interaction in the system. Sequence diagrams are the most popular diagram for dynamic modeling, which focuses on identifying the behavior within the system (Greenyer et al., 2013).

The result of business process modeling is shown by Figure 1. Figure 1 is the main business process in virtual collaboration in the jamu supply chain. The two main processes are raw

Table 1.

System Analysis Result
Analysis Results

All variables related to quality and quantity of products and raw materials including transportation aspect and cost All type of jamu products

Jamu production

Seasonal, bulky, and perishable products

Farmers, wholesalers, industry, SME

Control quality

Herbal material

Target quantity and quality 
material entity and processing entity. Wholesaler inputs raw material data and the system sends it to the processor. The processor will check the demand forecasting, make a production, and purchasing planning. The "+" sign in the diagram describes the decomposed process. It means the process has detailed processes. Each process is detailed in the next diagram.

\subsection{Design class diagram and database of the system}

Class diagram is an UML diagram type, describes the structure of a system by showing the system's classes, their attributes, operations (or methods), and the relationships among objects (Herchi and Abdessalem, 2012). It is used both for general conceptual modeling of the systematics of the application, and for detailed modeling translating the models into programming code. The class diagram is used to model each sub system entity in virtual collaboration system and generate data model of the system. After data design is generated, a NoSQL (Not only SQL) database is designed by using Java Script Object Notation (JSON). The structure of this database is graph database form to represent virtual interaction in the system.

Each entity in the system is modeled into class diagram. The class diagram of the system is shown in Figure 2. All processing and purchasing class require input raw material and sales of products. The relationship between actors is generated into graph data where data in an actor or entity send to other actor for collaboration purposes. We also generate the class diagram into object oriented java programming.

The example design of graph data for a direct and indirect purchasing using firebase cloud database is described in Figure 3.

\subsection{Problem formulation and mathematical modeling}

We describe the problem description into goal programming mathematical model. The goal programming represents multigoal decision approach of the system. Goal programming is a branch of multiobjective optimization, which in turn is a branch of multicriteria decision analysis (MCDA) (Romero, 2014). As satisfaction of the target is deemed to satisfy the decision maker(s), an underlying satisficing philosophy is assumed. According to the problem description the objective constraints for goal programming are

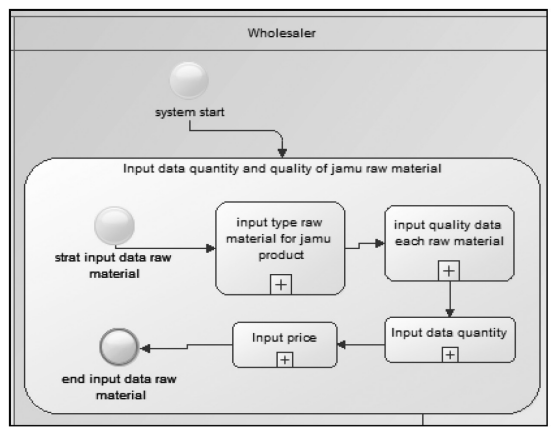

(a)

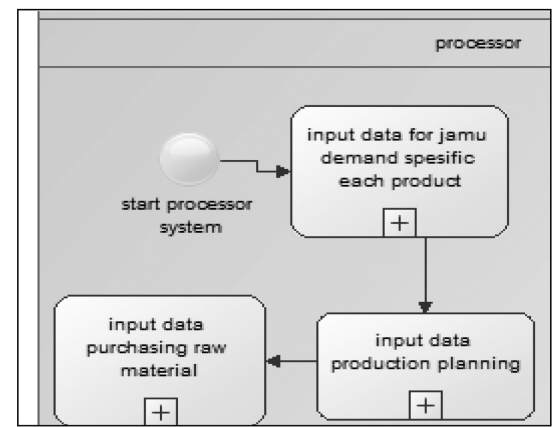

(b)
Analysis and Design of Virtual

Collaboration

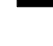


Proceedings of

MICoMS 2017

\section{2}

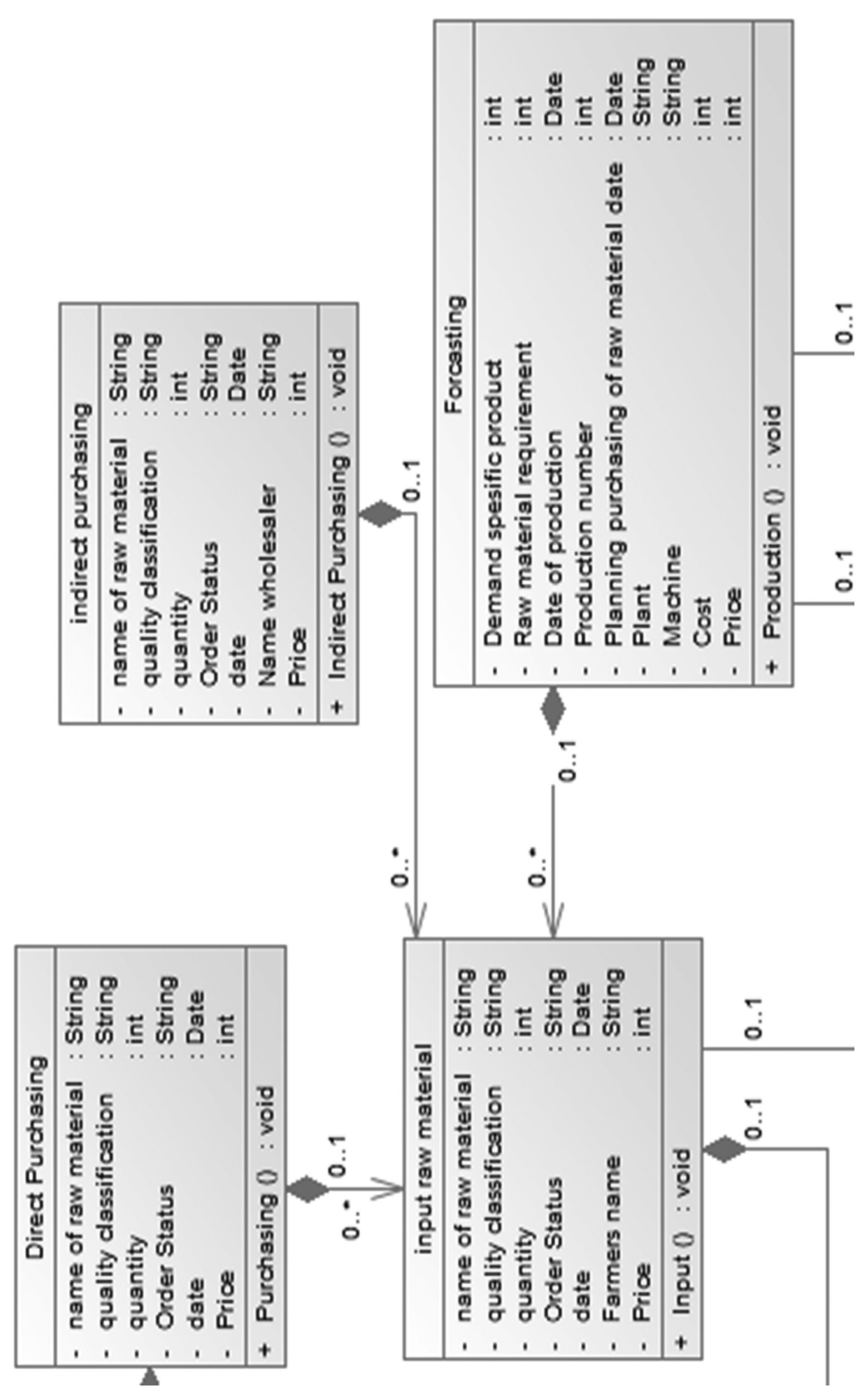

Figure 2.

Main Class Diagram of the System 
third-zephyr-130523

I-... Virtual Collaboration Supply Chain

$\frac{1}{-1}$-.. Direct Purchasing

[..... Buyer: "no assigned buye।

date: "01 August 201;

.... name of raw material: "Kunyit

..... order status: " unassigned

price: $1500 \theta \theta$. $x$

.... quality classification: "A "

quantity: $10 €$

-1... Indirect Purchasing

L.... Name of raw material: "Kunir"

Quality classification: "B"

-... buyer: "PT A'

date: "01 August 201;

order status: " assigned

price: 750006

quantity: $5 \epsilon$

$$
\sum_{i=1}^{I} \sum_{j=1}^{J} X_{i j}+\sum_{i=1}^{I} \sum_{k=1}^{K} X_{i k}+d_{1}^{-}-d_{1}^{+}=T^{q p}
$$$$
\sum_{s=1}^{S} \sum_{j=1}^{J} M_{s \rightarrow j}+\sum_{f=1}^{F} \sum_{j=1}^{J} M_{f \rightarrow j}+d_{2}^{-}-d_{2}^{+}=T^{q t p}
$$

$$
\sum_{S=1}^{S} \sum_{j=1}^{J} Q_{S \rightarrow j}^{b b}+\sum_{f=1}^{F} \sum_{j=1}^{J} Q_{f \rightarrow j}^{b b}+d_{3}^{-}-d_{3}^{+}=T^{q q b b}
$$

Analysis and

Design of

Virtual

Collaboration

\section{3}

Figure 3.

Example Graph Data

of Virtual

Collaboration System

\begin{tabular}{|c|c|c|c|}
\hline Run & Variables (Total) & Value in Contract & \\
\hline \multirow[t]{10}{*}{1 (First contract) } & Quantity material A & 11.2 ton & \\
\hline & Quantity material B & 10.9 ton & \\
\hline & Quantity material C & 12.5 ton & \\
\hline & Quantity material D & 12 ton & \\
\hline & Quality material A & $98.6 \%$ & \\
\hline & Quality material B & $99.1 \%$ & \\
\hline & Quality material C & $98.2 \%$ & \\
\hline & Quality material D & $99.2 \%$ & Table 2. \\
\hline & Transportation cost & Rp 14500100 & Result from the \\
\hline & Production cost & Rp 102900000 & Example Case \\
\hline
\end{tabular}

in Jamu Supply Chain 


$$
\sum_{s=1}^{S} \sum_{j=1}^{J} Q_{i \rightarrow j}^{\not p}+\sum_{f=1}^{F} \sum_{j=1}^{J} Q_{i \rightarrow k}^{\not p}+d_{4}^{-}-d_{4}^{+}=T^{q q p}
$$

Equation (1) is the goal of quantity product that is produced by industry and small medium enterprise (SME). It is defined by summation of product $I$ produced by industry $I$ and small medium enterprise $k$. The Equation (2) is the goal of quantity raw materials that are transported from farmers and(or) wholesaler into industry and(or) SME. It is defined by summation of total raw material from farmer $f$ to industry $j$ and summation of raw material from wholesaler $s$ to industry $j$. The Equations (3) and (4) are goals to meet the quality of the raw material and product in order to fulfill customer request. It is summation of quality of the product $i$ produced in industry $j$ and SME $k$.

And availability logistic transportation for transport raw material in Eq. (9) as follows

$$
\sum_{s=1}^{S} \sum_{j=1}^{J} M_{s \rightarrow j}+\sum_{f=1}^{F} \sum_{j=1}^{J} M_{f \rightarrow j} \leq T^{q t p}
$$

Also the prediction of availability raw material with specific quality for production purpose as described by Eqs. (10) and (11)

$$
\begin{aligned}
& \sum_{s=1}^{S} \sum_{j=1}^{J} Q^{b b} Q_{s \rightarrow j}^{b b}+\sum_{f=1}^{F} \sum_{j=1}^{J} Q^{b b} Q_{f \rightarrow j}^{b b} \leq T Q^{q q b b} \\
& \sum_{s=1}^{S} \sum_{j=1}^{J} Q^{p p} Q_{i \rightarrow j}^{p p}+\sum_{f=1}^{F} \sum_{j=1}^{J} Q^{\not p} Q_{i \rightarrow k}^{p p} \leq T Q^{q q p}
\end{aligned}
$$

We design contract value for quantity and quality for supply chain actor collaboration in partial approach by integrating backpropagation algorithm and then use goal approach as multicriterial decision approach. We generate data for simulation purposes follow the real case data trend for three adaptive fair contracts. According to the implementation of goal programming formulation and backpropagation algorithm with Neuroph API in java programming we got the estimated results from real case problem. We also present total quantity material and quality in the supply chain. We present the total cost for production and transportation. The result is obtained by integrating neural network and goal programming. We got neural network with input from quality and quantity of raw material and product for each supply chain actor. The training phase for two times running got neural network with hidden layer 17 and error 0.004198 .

\section{Conclusion and future research}

In this paper, we design adaptive contract system in jamu supply chain using system analysis and design, goal programming formulation, and backpropagation neural network to predict the uncertainty variable in the system. According to the result our system can determine the variables value in contract system with three times running to design three contract according to the availability and quality of raw material and jamu product. Although our system is validated and verified as a system, this model is initial research in adaptive contract for agricultural supply chain that need to determine other aspects such as the performance of supply chain, government regulation, product logistic, and organization structural in contract system. We 
recommend the future research to expand this initial research into other aspects, hence the contract design is comprehensive in order to increase the supply chain system performance.

\section{References}

Almutairi, S., Abu-Samaha, A., Bell, G., \& Chen, F. (2013), "An enhanced use case diagram to model Context Aware Systems". In Proceedings IEEE Science and Information Conference, pp. 270-274.

Arshinder, K., Kanda, A. and Deshmukh, S.G. (2011). "A Review on Supply Chain Coordination: Coordination Mechanisms, Managing Uncertainty and Research Directions". In T.M. Choi, T. Cheng, Supply Chain Coordination under Uncertainty, Springer, Berlin, pp. 39-82.

Brenner, C.,Greenyer, J. and La Manna, V.P. (2013). "The ScenarioTools Play-Out of Modal SequenceDiagram Specifications with Environment Assumptions". Electronic Communications of theEASST, Vol. 58, pp.1-15.

Demuth, H.B., Beale, M.H., De Jess, O. and Hagan, M.T. (2014). "Neural Network Design”. Neural Network Design, 2nd edition, Martin Hagan, USA.

Elfahmi, K.R., Bos, R., Kayser, O., Woerdenbag, H.J. and Quax, W.J. (2008). Jamu: The Indonesian Traditional Herbal Medicine. Penerbit Eisei, Jakarta, pp. 14-34.

Greenyer, J., Brenner, C. and La Manna, V.P. (2013). “The ScenarioTools Play-Out of Modal Sequence Diagram Specifications with Environment Assumptions". Electronic Communications of the EASST, Vol. 58.

Herchi, H. and Abdessalem, W.B. (2012). "From User Requirements to UML Class Diagram". arXiv Preprint, arXiv:1211.0713.

Ho, C.J., Slivkins, A. and Vaughan, J.W. (2013). “Adaptive contract design for crowdsourcing”. Working Paper, Preliminary version to appear in the NIPS'13 Workshop on Crowdsourcing: Theory, Algorithms, and Applications; pp.1-21.

Ho, C.J., Slivkins, A. and Vaughan, J.W. (2016). "Adaptive Contract Design for Crowdsourcing Markets: Bandit Algorithms for Repeated Principal-Agent Problems”. Journal of Artificial Intelligence Research, Vol. 55, pp. 317-359.

Romero, C. (2014). "Handbook of Critical Issues in Goal Programming". Pergamon Press, Elsevier, United Kingdom.

Schroeder, A., Bauer, S. S., and Wirsing, M. (2011). “A Contract-Based Approach to Adaptivity”. Journal of Logic and Algebraic Programming, Vol. 80, No. 3-5, pp. 180-193.

Sutanto, J., Tan, C.H., Battistini, B. and Phang, C.W. (2011). "Emergent Leadership in Virtual Collaboration Settings: A Social Network Analysis Approach”. Long Range Planning, Vol. 44, No. 5, pp. 421-439.

Wasson, C.S. (2015). "System Engineering Analysis, Design, and Development: Concepts, Principles, and Practices". John Wiley \& Sons, Inc., Hoboken, New Jersey.

Yeung, W.K., Choi, T.M. and Cheng, T.C.E. (2011). "Supply Chain Scheduling and Coordination with Dual Delivery Modes and Inventory Storage Cost". International Journal of Production Economics, Vol. 132, No. 2, pp. 223-229.

Zur Muehlen, M. and Recker, J. (2013). "How Much Language is Enough? Theoretical and Practical Use of the Business Process Modeling Notation". In Seminal Contributions to Information Systems Engineering, Springer, Berlin, pp. 429-443.

\section{Corresponding author}

Aster Rakhmasari can be contacted at aster@kemenperin.go.id
Analysis and Design of Virtual Collaboration 